\title{
ANÁLISE DE ESTRATÉGIAS BASEADAS EM AGREGAÇÃO DOS RESERVATÓRIOS NO PLANEJAMENTO ENERGÉTICO DE SISTEMAS HIDROTÉRMICOS
}

\author{
Erico Da Silva Ventura*, LuCiana Martinez* \\ * Departamento de Engenharia Elétrica - Escola Politécnica, Universidade Federal da Bahia \\ Emails: ventura@ufba.br, lucianam@ufba.br
}

\begin{abstract}
This paper presents an analysis of differents strategies based on Stochastic Dynamic Programming (SDP) method with aggregation of reservoirs in a equivalent model for solution of hydroelectric operation scheduling problem, using in each of this strategies a combination of a model to represent stochaticity of equivalent model inflow energy and a different component of equivalent model to be taken as control variable. Based on this strategies implementations, the results achieved of simulations taking into account the operation of the hydroelectric plants of of two subsystems of brazilian system, are compared in order to analyzing the influence of each strategy used on electrical generation regularity and level of stored energy of subsystems.
\end{abstract}

Keywords- Stochastic Dynamic Programming, Optimal Scheduling, Reinforcement Learning, Hydrothermal Power Systems, Optimization.

Resumo - Este trabalho apresenta uma análise de diferentes estratégias baseadas na Programação Dinâmica Estocástica (PDE) com a agregação dos reservatórios em um modelo equivalente, para solução do problema do planejamento da operação energética de sistemas hidrotérmicos de geração, utilizando em cada uma dessas estratégias, uma combinação de um modelo para representação da estocasticidade da energia total afluente ao sistema equivalente e de um de seus componentes que é considerado como variável de controle. Com base na implementação dessas estratégias, os resultados obtidos nas simulações da operação de dois subsistemas do sistema brasileiro são comparados com o intuito de analisar a influência que o uso de cada uma dessas estratégias tem, ao longo do período de planejamento, na regularidade da geração de energia elétrica e nos níveis de energia armazenada de cada subsistema.

Keywords - Programação Dinâmica Estocástica, Planejamento Energético, Aprendizagem por Reforço, Sistemas Hidrotérmicos de Potência, Otimização.

\section{Introdução}

O objetivo do planejamento da operação energética de sistemas hidrotérmicos de geração de energia elétrica é obter metas de operação que minimizem o custo da energia elétrica produzida, além de garantir a qualidade e segurança no atendimento da demanda. O planejamento e a operação de um sistema hidrotérmico de geração correspondem a um problema de otimização cujo objetivo é reduzir os custos ao longo do horizonte de planejamento com a produção complementar de energia a partir de recursos não hídricos - como a geração térmica ou a importação de mercados vizinhos - e com o déficit de energia (racionamento), promovendo o aproveitamento racional dos recursos hídricos disponíveis (Ventura and Martinez, 2012).

Uma meta ótima de operação deve adotar, para cada estágio do planejamento, uma decisão que além de minimizar os custos imediatos, também mantenha o nível do reservatório em um estado que não comprometa a geração de energia elétrica, nem acarrete custos indesejáveis para os estágios futuros do planejamento, atendendo a demanda de forma econômica e confiável (Ventura and Martinez, 2012).

O problema de planejamento energético da operação de sistemas hidrotérmicos de geração é complexo devido a vários aspectos de modelagem, tais como a incerteza das vazões afluentes às usi- nas hidroelétricas do sistema, a interconexão de usinas em cascata e a não linearidade das funções de produção hidráulica e de custo de complementação (Ventura and Martinez, 2012). No caso do sistema brasileiro esta complexidade é acentuada, uma vez que é composto por grandes bacias interligadas e um grande número de reservatórios com capacidade de regularização plurianual.

A Programação Dinâmica Estocástica (PDE) é uma técnica de aprendizagem por reforço que incorpora em sua formulação aspectos estocásticos e não lineares do problema e é amplamente aplicada no problema do planejamento energético da operação de sistemas hidrotérmicos de geração. No entanto, a principal limitação desse método é a chamada "maldição da dimensionalidade", já que o esforço computacional cresce exponencialmente à medida que o número de variáveis de estado do problema aumenta, o que ocorre por exemplo com o aumento do número de usinas hidroelétricas consideradas no problema.

Uma das alternativas para tentar superar esta limitação da PDE é a técnica de agregaçãodesagregação dos reservatórios, que se baseia na representação simplificada de todo o sistema hidrotérmico em um modelo equivalente composto por um único reservatório equivalente de energia, o que reduz drasticamente o espaço dimensional do sistema. O reservatório equivalente baseia-se na estimação da energia elétrica produzida com o completo esvaziamento de todos os reservatórios 
do sistema a partir de um estado inicial de armazenamento. Sobre esse reservatório equivalente é aplicada a PDE para obtenção das metas de operação baseadas na representação composta dos reservatórios, de forma que, para aplicação dessas metas individualmente aos reservatórios do sistema, elas precisam ser posteriormente desagregadas, de acordo com uma regra de operação especificada (Jr., 1998),(Marcato, 2002),,(SerratCapdevila and Valdés, 2007),(Nandalal and Bogardi, 2007).

O objetivo deste trabalho é comparar políticas de operação obtidas a partir da PDE com o uso de um modelo equivalente para representação do sistema, com algumas estratégias que consideram diferentes combinações de variáveis de estado e decisão para o problema. São consideradas as representações da estocasticidade da energia total afluente ao sistema por um Modelo Markoviano - sendo as variáveis de estado neste caso representadas pela energia armazenada nos reservatórios no início do estágio e a energia total afluente ao sistema no estágio anterior - e por um modelo estocástico não correlacionado ou independente, cuja única variável de estado é representada pela energia armazenada nos reservatórios. As diferentes variáveis de decisão consideradas são o nível de energia armazenada nos reservatórios no início do estágio seguinte e a quantidade de energia defluente do sistema em cada estágio.

As quatro diferentes estratégias, resultantes das combinações possíveis dos modelos estocásticos com as variáveis de decisão, foram comparadas com base nos resultados das simulações da operação de dois subsistemas do sistema brasileiro, analisando-se para cada estratégia a regularidade da geração de energia elétrica e o comportamento do nível do energia armazenada nos reservatórios.

\section{Formulação do Problema de Planejamento da Operação Energética}

O problema de planejamento da operação energética de sistemas hidrotérmicos de geração de energia elétrica, em sua versão determinística e considerando que o sistema é representado por um modelo equivalente, pode ser formulado como o seguinte problema de programação não linear:

$$
\min \sum_{t=1}^{T} \psi_{t}\left(d_{t}-e d_{t}\right)+V\left(e_{T}\right)
$$

sujeito a:

$$
\begin{gathered}
e_{t+1}=e_{t}+e a_{t}-e d_{t} \\
\underline{e a_{t}} \leq e a_{t} \leq \overline{e a_{t}} \\
\underline{e d_{t}} \leq e d_{t} \leq \overline{e d_{t}} \\
e v_{t} \geq 0 \\
e a_{1} \text { dado }
\end{gathered}
$$

$$
\forall t, t=1,2, \ldots, T
$$

A função objetivo (1) é composta pelo custo operacional durante o período de planejamento e o custo futuro associado ao estado final de armazenamento do reservatório.

O custo operacional $\psi_{t}(\cdot)$ representa o custo de geração complementar a partir de recursos não hidrícos. Como conseqüência da otimização desses recursos, $\psi_{t}$ é uma função convexa crescente da geração complementar e depende da demanda $d_{t}$ a ser atendida.

A função $V(\cdot)$ representa o custo futuro associado ao estado da energia armazenada nos reservatórios ao final do período de planejamento $T$. O objetivo desta condição terminal é equilibrar os custos operacionais decorrentes do uso da água ao longo do período de planejamento com os custos futuros após esse período.

A equação de igualdade (2) representa o balanço da energia armazenada $e_{t}$ nos reservatórios do sistema, onde $e a_{t}$ é a energia total afluente ao sistema e $e d_{t}$ é a quantidade de energia defluente do sistema no estágio $t$. Termos referentes às perdas de energia devido à evaporação e infiltração não são considerados por questão de simplicidade. Cada um desses valores de energia do sistema são calculados com base na estimação das produtibilidades das usinas do sistema (Jr., 1998),(Marcato, 2002).

Os limites superiores e inferiores das variáveis, representados pelas equações (3)-(5), são impostos por consequência das restrições operacionais das usinas hidroelétricas e por outras restrições associadas ao uso múltiplo da água, como controle de cheias, irrigação ou navegação. O termo $e v_{t}$ presente na restrição (5) é a energia vertida que é perdida por conta da necessidade de vertimento de uma usina por não poder ser estocada ou absorvida pelo mercado.

\section{Programação Dinâmica}

Seja um sistema dinâmico discreto no tempo representado como (Bertsekas, 2005):

$$
e_{t+1}=f_{t}\left(e_{t}, e d_{t}, e a_{t}\right) \quad t=1,2, \ldots, T
$$

Seja o custo funcional associado ao sistema (7) definido como $c_{t}\left(e_{t}, e d_{t}, e a_{t}\right)$, de forma que o custo ocorrido no estágio $k$ acumula-se sobre o tempo e o custo total do sistema seja dado por:

$$
\mathrm{C}_{\text {Total }}=\sum_{t=1}^{T} c_{t}\left(e_{t}, e d_{t}, e a_{t}\right)+c_{T}\left(e_{T}\right)
$$

onde $c_{T}\left(e_{T}\right)$ representa o custo ocorrido no final do processo. Devido à presença da variável $e a_{t}$, geralmente o custo é uma variável aleatória.

Dado um estado inicial de energia armazenada do sistema, $e a_{1}$, uma política ótima para atuar em 
(7) pode ser determinada através da solução do seguinte problema:

$$
\begin{aligned}
J(e d)=\min _{e d_{t}} E_{e a_{t}}\left\{\sum_{t=1}^{T} c_{t}\left(e_{t}, e d_{t}, e a_{t}\right)+c_{T}\left(e_{T}\right)\right\} \\
s . a:\left\{\begin{array}{l}
e_{t+1}=f_{t}\left(e_{t}, e d_{t}, e a_{t}\right) \\
e d_{t} \subset E D_{t} \\
t=1,2, \ldots, T
\end{array}\right.
\end{aligned}
$$

onde $E_{e a_{t}}$ representa a esperança matemática do custo em relação à distribuição da variável aleatória $e a_{t}$ envolvida no problema.

\subsection{Estratégias definidas para PDE no Planeja- mento Energético}

As quatro estratégias analisadas neste trabalho foram definidas através da combinação de um dos dois modelos estocásticos considerados com o uso de uma das duas possíveis variáveis que podem ser tomadas como variável de decisão. Com relação à representação estocástica da energia total afluente ao sistema, foram consideradas as seguintes alternativas:

(i) O processo estocástico da variável aleatória associada à energia total afluente ao sistema, é representado por um modelo Periódico Autorregressivo de ordem 1, PAR(1), ou seja, considera-se que a energia total afluente no estágio $t$ depende somente da energia total afluente em $t-1$, e neste caso, as variáveis de estado são representadas além do nível de energia armazenada nos reservatórios do sistema no começo de cada estágio $e_{t}$ - também pela energia total afluente ao sistema no estágio anterior, $e a_{t-1}$.

(ii) O processo estocástico da variável aleatória associada à energia total afluente ao sistema, é considerado independente no tempo ou não correlacionado. Neste caso a PDE terá apenas a variável de estado $e_{t}$.

Em geral, a variável de decisão que é comumente adotada no problema do planejamento energético é a defluência $u_{t}$, que está associada à quantidade de água liberada do reservatório durante o estágio $t$ e é composta pela quantidade turbinada, $q_{t}$, e vertida, $s_{t}$. Com o uso da representação equivalente do sistema, essa variável de decisão passa a ser dada pela energia defluente, que é a energia gerada a partir dessa quantidade de água que é liberada do reservatório.

A variável de decisão da PDE pode também ser representada pelo nível do reservatório no início do estágio seguinte $x_{t+1}$ (Nandalal and Bogardi, 2007), ou no caso da representação equivalente, pelo nível da energia armazenada no início do estágio seguinte, dada por $e_{t+1}$.

Em cada estágio, as decisões ótimas são determinadas através da minimização da soma do custo presente mais o custo futuro esperado. Este custo é aditivo no sentido que o custo ocorrido em um estágio $t$ qualquer acumula-se sobre o tempo. A técnica de resolução utilizada é conhecida como backward, pois a busca da solução ótima é iniciada do estágio final $T$ e segue até o estágio inicial.

A seguir, são apresentadas as estratégias para PDE que foram utilizadas neste trabalho com suas respectivas equações recursivas (que correspondem à solução da PDE).

\section{Estratégia 1}

Uso do modelo markoviano para representação da estocasticidade da energia total alfuente e nível de energia armazenada em $t+1$ como variável de decisão.

Variáveis de estado: $\left(e_{t}, e a_{t-1}\right)$

Variável de decisão: $\left(e_{t+1}\right)$

$$
\begin{aligned}
& F_{t}\left(e_{t}, e a_{t-1}\right)=\min _{\left\{e_{t+1}\right\} \in \Omega_{t}}\left\{\psi_{t}\left(d_{t}-e d_{t}\right)+\right. \\
& \left.+E_{e a_{t} \mid e a_{t-1}}\left[F_{t+1}\left(e_{t+1}, e a_{t}\right)\right]\right\} \\
& \text { sujeito } a:\left\{\begin{array}{l}
F_{T}\left(e_{T}, e a_{T-1}\right)=V\left(e_{T}\right) \\
e d_{t}=\left(e_{t}+e a_{t}\right)-e_{t+1} \\
\forall t, t=T, T-1, \ldots, 1
\end{array}\right.
\end{aligned}
$$

\section{Estratégia 2}

Uso do modelo não correlacionado para representação da estocasticidade da energia total alfuente e nível de energia armazenada em $t+1$ como variável de decisão. Variáveis de estado: $\left(e_{t}\right)$

Variável de decisão: $\left(e_{t+1}\right)$

$$
\begin{array}{r}
F_{t}\left(e_{t}\right)=\min _{\left\{e_{t+1}\right\} \in \Omega_{t}}\left\{\psi_{t}\left(d_{t}-e d_{t}\right)+\right. \\
\left.+E_{e a_{t}}\left[F_{t+1}\left(e_{t+1}\right)\right]\right\} \\
\text { sujeito } a:\left\{\begin{array}{l}
F_{T}\left(e_{T}\right)=V\left(e_{T}\right) \\
e d_{t}=\left(e_{t}+e a_{t}\right)-e_{t+1} \\
\forall t, t=T, T-1, \ldots, 1
\end{array}\right.
\end{array}
$$

\section{Estratégia 3}

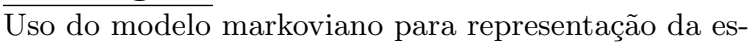
tocasticidade da energia total alfuente e energia defluente no estágio como variável de decisão.

Variáveis de estado: $\left(e_{t}, e a_{t-1}\right)$

Variável de decisão: $\left(e d_{t}\right)$

$$
\begin{aligned}
& F_{t}\left(e_{t}, e a_{t-1}\right)=\min _{\left\{e d_{t}\right\} \in \Omega_{t}}\left\{\psi_{t}\left(d_{t}-e d_{t}\right)+\right. \\
& \left.+E_{e a_{t} \mid e a_{t-1}}\left[F_{t+1}\left(e_{t+1}, e a_{t}\right)\right]\right\} \\
& \text { sujeito } a:\left\{\begin{array}{l}
F_{T}\left(e_{T}, e a_{T-1}\right)=V\left(e_{T}\right) \\
\forall t, t=T, T-1, \ldots, 1
\end{array}\right.
\end{aligned}
$$

\section{Estratégia 4}

Uso do modelo não correlacionado para representação da estocasticidade da energia total alfuente e energia defluente no estágio como variável de decisão.

Variáveis de estado: $\left(e_{t}\right)$

Variável de decisão: $\left(e d_{t}\right)$

$$
\begin{aligned}
F_{t}\left(e_{t}\right)=\min _{\left\{e d_{t}\right\}} \in \Omega_{t}\left\{\psi_{t}\left(d_{t}-e d_{t}\right)+\right. \\
\left.+E_{e a_{t}}\left[F_{t+1}\left(e_{t+1}\right)\right]\right\}
\end{aligned}
$$




$$
\text { sujeito } a:\left\{\begin{array}{l}
F_{T}\left(e_{T}\right)=V\left(e_{T}\right) \\
\forall t, t=T, T-1, \ldots, 1
\end{array}\right.
$$

onde $F_{t}\left(e_{t}\left[, e a_{t-1}\right]\right)$ representa o valor mínimo de operação esperado desde o estágio $t$ até o final do horizonte $T$, supondo que o sistema se encontra no estado $\left(e_{t}\left[, e a_{t-1}\right]\right)$ e, em função da decisão tomada, transita para o estado $\left(e_{t+1}\left[, e a_{t}\right]\right)$ com $\Omega_{t}$ sujeito às restrições dadas pelas equações (2)(6).

\subsection{Tratamento da estocasticidade da energia to- tal afluente}

Seja o histórico de energias totais afluentes mensais denotado pelo processo estocástico $e a_{t(r, m)}$, com $t(r, m)=12(r-1)+m$, onde $r=1, \ldots, n$ representa o número de anos no histórico e $m=$ $1, \ldots, 12$ representa os meses do ano. O processo estocástico $e a_{t(r, m)}$ é sazonal, com período de 12 meses. A média sazonal desse processo é denotada por $\mu_{m}$, e sua variância sazonal é denotada por $\sigma_{m}^{2}$, sendo estimadas como:

$$
\begin{gathered}
\widehat{\mu}_{m}=\frac{1}{n} \sum_{r=1}^{n} e a_{t(r, m)} \\
\widehat{\sigma}_{m}^{2}=\frac{1}{(n-1)} \sum_{r=1}^{n}\left(e a_{t(r, m)}-\widehat{\mu}_{m}\right)^{2}
\end{gathered}
$$

Um processo livre de sazonalidade na média e na variância pode ser extraído do histórico de energias totais afluentes da seguinte forma:

$$
z_{t(r, m)}=\frac{e a_{t(r, m)}-\widehat{\mu}_{m}}{\widehat{\sigma}_{m}}
$$

O processo $z_{t(r, m)}$ apresenta a mesma natureza estocástica do processo $e a_{t(r, m)}$. Uma hipótese importante considerada na construção dos modelos estocásticos para energias totais afluentes é a hipótese de estacionariedade do processo $z_{t(r, m)}$, a qual afirma que a distribuição de probabilidade conjunta:

$$
p\left(z_{t(1,1)}, \ldots, z_{t(1,12)}, \ldots, z_{t(r, 1)}, \ldots, z_{t(r, 12)}\right)
$$

não se altera para qualquer $r \geq 1$.

Considerando que o processo $z_{t(r, m)}$ satisfaz um modelo $\operatorname{PAR}(1)$,tem-se que:

$$
z_{t(r, m)}=\phi_{m, 1} z_{t(r, m)-1}+a_{t(r, m)}
$$

sendo $\phi_{m, 1}$ uma função periódica com período de 12 meses e $a_{t(r, m)}$ é um processo estocástico com $E\left(a_{t(r, m)}\right)=0, E\left(a_{t(r, m)} a_{t(r, m)+k}\right)=0$ se $k \neq 0$ e $E\left(a_{t(r, m)}^{2}\right)=\widehat{\tau}_{m}^{-1}=\sigma_{a, m}^{2}$. Assume-se que $a_{t(r, m)}$ é $N\left(0, \widehat{\tau}_{m}^{-1}\right)$. Os parâmetros $\phi_{m, j}$ e $\widehat{\tau}_{m}^{-1}$ foram estimados pelo método de máxima verossimilhança.

Dessa forma, $\widehat{e a}_{t(r, m)}$ é representada como:

$$
\widehat{e a}_{t(r, m)}=\widehat{\mu}_{m}+\frac{\widehat{\sigma}_{m}}{\widehat{\sigma}_{m-1}} \phi_{m, 1}\left(\widehat{e a}_{t(r, m)-1}-\widehat{\mu}_{m-1}\right)
$$

\section{Considerações sobre a implementação da PDE}

A representação equivalente do sistema utilizada neste trabalho adotou a regra linear para operação dos reservatórios, supondo assim essa operação paralelamente no mesmo percentual de armazenamento. Foram utilizadas parábolas de correção para correção das energias controlável, de vazão mínima e de vazão máxima, em função da energia armazenada (Marcato, 2002).

Para o ajuste da distribuição de probabilidades associadas às energias totais afluentes, bem como para a determinação das suas faixas utilizadas na otimização, foi utilizado um histórico de energia total afluente calculado a partir de uma série mensal de vazões de 77 anos (1931-2008) (ONS, 2013) para as usinas dos dois subsistemas considerados. O custo futuro associado ao último estágio é considerado nulo, e um horizonte de 10 anos (120 estágios) é utilizado pelo método, com o intuito de tornar desprezível a influência desse custo futuro nulo nos primeiros estágios de planejamento.

A demanda de geração para todos os estágios foi assumida igual à soma das potências instaladas das usinas de cada susbsistema. As variáveis de energia defluente e energia armazenada foram discretizadas em 100 valores. Os valores mínimo e máximo para a energia armazenada são respectivamente 0 e a energia armazenável máxima do reservatório para o mês corrente. Os valores mínimo e máximo para a energia defluente são respectivamente a energia de vazão mínima e a energia de vazão máxima, que é o máximo de energia que pode ser gerada, limitada pelo engolimento máximo da usina, as quais são calculadas com base nas restrição operacional das usinas. A variável de estado associada às energias totais afluentes transformadas foi discretizada em 10 faixas.

O custo operacional $\psi_{t}$ é, em geral, obtido pelo despacho ótimo de geração não hidráulica. Para o aspecto não linear do sistema brasileiro, uma estimativa do custo operacional é dada pela função quadrática:

$$
\psi_{t}=\left(d_{t}-e d_{t}\right)^{2}
$$

A partir da tabela de decisões ótimas obtida através da PDE, uma simulação da operação do subsistema é realizada baseada em valores reais do histórico de vazões afluentes ao sistema, no período compreendido entre 1931 até 2008. O simulador utilizado desagrega as metas obtidas em função do níveis dos reservatórios das usinas, garantindo que as restrições envolvidas no problema não sejam violadas. 


\section{Resultados Obtidos}

Nos estudos realizados foram considerados dois subsistemas: o subsistema da bacia do rio Grande e o subsistema da bacia do rio Paranaíba. Esses subsistemas são ilustrados na figura 1 e suas principais características são apresentadas na tabela 1.

Tabela 1: Dados dos subsistemas considerados.

\begin{tabular}{|c|c|c|}
\hline $\begin{array}{c}\text { Características dos } \\
\text { Subsistemas (MW) }\end{array}$ & $\begin{array}{c}\text { Subsistema do } \\
\text { Rio Grande }\end{array}$ & $\begin{array}{c}\text { Subsistema do } \\
\text { Rio Paranaíba }\end{array}$ \\
\hline $\begin{array}{c}\text { Capacidade } \\
\text { Instalada }\end{array}$ & 7312,2 & 5790 \\
\hline $\begin{array}{c}\text { Energia Armazenável } \\
\text { Máxima }\end{array}$ & 30596,64 & 23141,44 \\
\hline $\begin{array}{c}\text { Energia } \\
\text { Defluente min/max }\end{array}$ & $936,55 / 6450$ & $488,59 / 5900,36$ \\
\hline
\end{tabular}

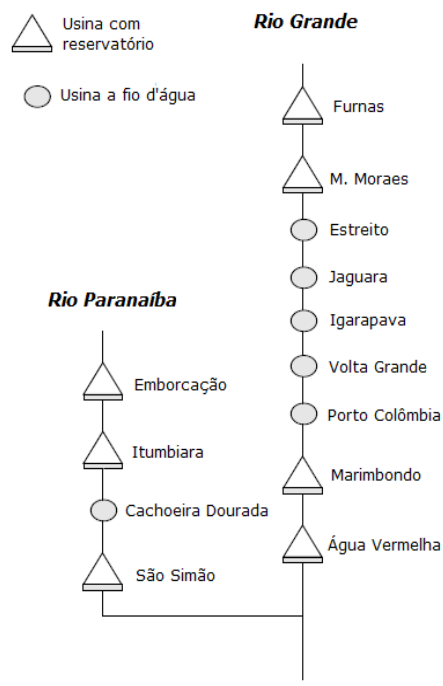

Figura 1: Cascata com os subsistemas considerados.

A tabela 2 apresenta a média, desvio padrão e o total da geração hidroelétrica obtidos nas simulações dos dois subsistemas, para cada estratégia definida na seção 3.1 deste trabalho.

As maiores médias de geração hidroelétrica e totais de energia gerados em todo período considerado são obtidos com o modelo Markoviano combinado com a energia defluente como variável de decisão (estratégia 3), que é a estratégia comumente usada nas modelagens do problema do planejamento energético. Apesar da estratégia que combina um modelo estocástico não correlacionado e a energia defluente como variável de decisão (estratégia 4) ter apresentado o menor desvio padrão nas simulações, esta estratégia mantém o nível de energia armazenada muito abaixo em relação às demais estratégias - conforme pode ser
Tabela 2: Estatísticas de geração dos subsistemas.

\begin{tabular}{|c|c|c|c|}
\hline \multirow{2}{*}{ Estratégia } & $\begin{array}{c}\text { Geração } \\
\text { Média } \\
(\mathrm{MW})\end{array}$ & $\begin{array}{c}\text { Desvio } \\
\text { Padrão } \\
(\mathrm{MW})\end{array}$ & $\begin{array}{c}\text { Total } \\
\text { Gerado } \\
(\mathrm{MW})\end{array}$ \\
\hline \multicolumn{4}{|c|}{ Subsistema do Rio Grande } \\
\hline PAR(1)/e $e_{t+1}$ & 4247,18 & 1623,87 & 3924396,90 \\
INDEPENDENTE $/ e_{t+1}$ & 4256,29 & 1602,67 & 3932810,83 \\
PAR(1)/ed & 4256,53 & 1551,33 & 3933037,22 \\
INDEPENDENTE $/ e d_{t}$ & 4184,65 & 1353,16 & 3866616,48 \\
\hline \multicolumn{3}{|c|}{ Subsistema do Rio Paranaíba } \\
\hline PAR $(1) / e_{t+1}$ & 3268,80 & 1002,48 & 3020367,97 \\
INDEPENDENTE $/ e_{t+1}$ & 3287,22 & 1050,02 & 3037396,74 \\
PAR $(1) / d_{t}$ & 3290,76 & 990,71 & 3040665,57 \\
INDEPENDENTE $/ d_{t}$ & 3224,39 & 818,53 & 2979332,79 \\
\hline
\end{tabular}

observado na figura 3 - o que diminui a produtividade das usinas e pode comprometer o sistema, sobretudo em períodos caracterizados pela ocorrência de seqüências hidrológicas desfavoráveis, uma vez que tais períodos possuem maiores custos operacionais e maiores riscos de déficit, sendo por isso considerados os mais importantes do planejamento.

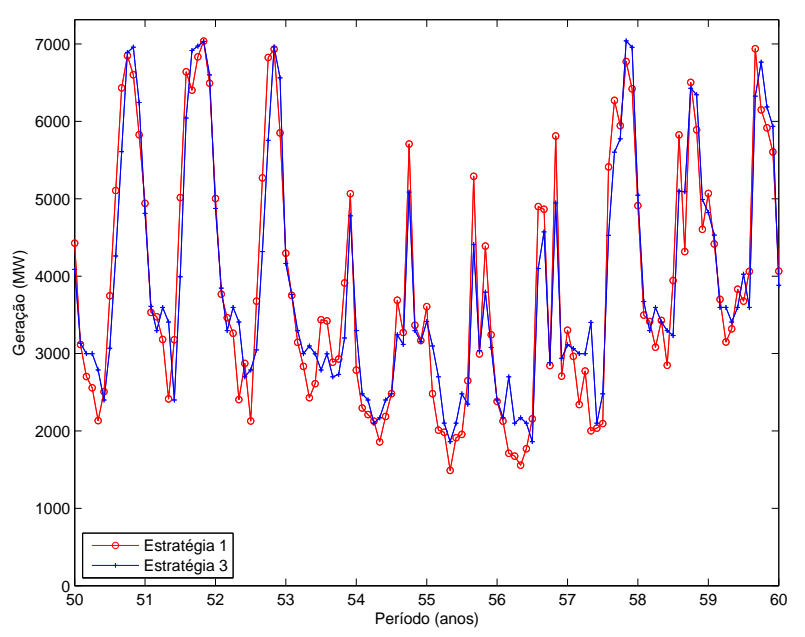

Figura 2: Geração obtida em simulações do subsistema do Rio Grande (década de 50).

A estratégia que combina o modelo Markoviano com a variável de decisão sendo a energia armazenada em $t+1$ (estratégia 1) apresentou resultados similares à estratégia 3 , embora com uma geração média ligeiramente menor, desvio padrão um pouco maior e com a média da energia armazenada ao longo do período simulado em torno de $7 \%$ mais baixa, porém com um desvio padrão significativamente menor do nível de energia armazenada, conforme pode ser observado nas figuras 2 e 3 , que mostram os resultados das simulações para o subsistema da bacia do Rio Grande no período de 1950 a 1960, caracterizado pela ocorrência de baixas afluências e do período crítico 
do sistema (1952 a 1956).

Observando a figura 3, nota-se que as estratégias baseadas em modelos estocásticos não correlacionados (estratégias 2 e 4) apresentam pouca precisão para previsão das energias totais afluentes, o que impede que em períodos mais secos o sistema possa fazer uso mais eficaz do recurso hídrico disponível, apresentando maiores oscilações nos níveis de energia armazenada (estratégia 4) ou na quantidade de energia gerada (estratégia 2). Apesar disso, a estratégia 2 mantém o nível médio de energia armazenado mais alto, denotando maior segurança do sistema no período crítico.

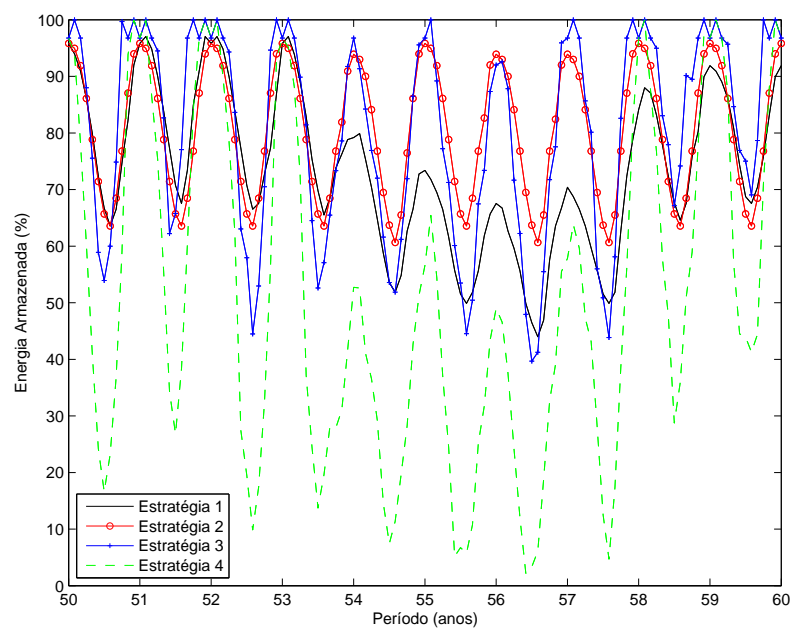

Figura 3: Energia armazenada para subsistema da bacia do Rio Grande - Década de 50.

Apesar de algumas diferenças encontradas nos resultados destas simulações baseadas na agregação de reservatórios, com relação às mesmas estratégias aplicadas à operação isolada das usinas (Ventura and Martinez, 2012), em geral os resultados obtidos para esses dois diferentes casos são similares, tendo como diferença significativa o caso da estratégia 1, que para representação agregada apresenta desempenho similar ao da estratégia 3, enquanto no caso da operação isolada das usinas, o desvio padrão significativamente maior da média de geração torna essa estratégia menos interessante que a estratégia 3 .

\section{Conclusões}

Este trabalho apresentou uma análise de quatro estratégias para PDE aplicada à solução do problema de planejamento energético, com diferentes modelos estocásticos e variáveis de decisão. $\mathrm{O}$ desempenho das abordagens foi analisado através de simulações realizadas para os subsistemas das bacias do Rio Grande e do Rio Paranaíba.

Os melhores desempenhos foram obtidos com as duas estratégias que utilizam o modelo estocástico Markoviano - as quais demonstraram resulta- dos similares - embora o uso da energia defluente como variável de decisão melhore a regularidade no atendimento à demanda e mantenha níveis de energia armazenada mais altos, sobretudo em períodos hidrológicos desfavoráveis. Embora o uso de um modelo não correlacionado combinado com a energia armazenada em $t+1$ como variável de decisão apresente desvio padrão da energia gerada um pouco mais alto, o comportamento da energia armazenada apresenta níveis médios mais elevados, sobretudo no período crítico, o que denota uma maior segurança do sistema.

\section{Agradecimentos}

Este trabalho contou com o suporte financeiro fornecido pela Fundação de Amparo à Pesquisa do Estado da Bahia (FAPESB) e pelo Conselho Nacional de Desenvolvimento Científico e Tecnológico $(\mathrm{CNPq})$.

\section{Referências}

Bertsekas, D. P. (2005). Dynamic Programming and Optimal Control, Vol. 1, 3 edn, Athena Scientific, Belmont, Massachusetts.

Jr., G. C. (1998). Modelo Equivalente Não Linear para o Planejamento da Operação a Longo Prazo de Sistemas de Energia Elétrica, Tese de Doutorado, FEEC-Unicamp, Campinas.

Marcato, A. L. M. (2002). Representação Híbrida de Sistemas Equivalentes e Individualizados para o Planejamento da Operação de Médio Prazo de Sistemas de Potência de Grande, Tese de Doutorado, Departamento de Engenharia Elétrica (DEE) - Pontifícia Universidade Católica do Rio de Janeiro (PUC-RIO), Rio de Janeiro.

Nandalal, K. D. W. and Bogardi, J. J. (2007). Dynamic Programming Based Operation of Reservoirs: Applicability and Limits, Cambridge University Press, New York. DOI: 10.1017/CBO9780511535710

ONS (2013). Operador Nacional do Setor Elétrico, http://www.ons.org.br, Rio de Janeiro - RJ, Brazil.

Serrat-Capdevila, A. and Valdés, J. (2007). An alternative approach to the operation of multinational reservoir systems: Application to the amistad \& falcon system (lower rio grande/r'10 bravo), Water Resources Manage-ment 21(4): 677-698. DOI: 10.1007/s11269-006-9035-1

Ventura, E. and Martinez, L. (2012). Análise de estratégias para programação dinâmica no problema de planejamento energético de sistemas hidrotérmicos, CBA 2012 - XIX Congresso Brasileiro de Automática . 